\title{
Short Time and Low Temperature Reaction between Metal Oxides through Microwave-Assisted Hydrothermal Method
}

\author{
S. M. V. Novais, P. C. R. Silva, Z. S. Macedo, and L. B. Barbosa \\ Advanced Ceramic Materials Group, Physics Department, Federal University of Sergipe, 49.100-000, São Cristóvão, SE, Brazil \\ Correspondence should be addressed to Z. S. Macedo; zelia.macedo@gmail.com
}

Received 4 September 2016; Revised 9 October 2016; Accepted 6 November 2016

Academic Editor: Veer P. S. Awana

Copyright ( 2016 S. M. V. Novais et al. This is an open access article distributed under the Creative Commons Attribution License, which permits unrestricted use, distribution, and reproduction in any medium, provided the original work is properly cited.

\begin{abstract}
This work demonstrates the possibility of synthesis of cadmium tungstate at low temperatures using oxide precursors. Cadmium tungstate $\left(\mathrm{CdWO}_{4}\right)$ scintillator was produced via microwave-assisted hydrothermal reaction using the precursors CdO and $\mathrm{WO}_{3}$. The methodology was based on microwave radiation for heating, which is remarkably faster than the solid-state route or conventional hydrothermal procedure. $\mathrm{CdWO}_{4}$ monoclinic (wolframite) structure was successfully obtained at $120^{\circ} \mathrm{C}$ for synthesis times as short as $20 \mathrm{~min}$. This route does not require the use of templates or surfactants and yields self-assembled nanorods with size of around $24 \pm 9 \mathrm{~nm}$ width and $260 \pm 47 \mathrm{~nm}$ length. The growth mechanism for the formation of $\mathrm{CdWO}_{4}$ involves microwaveinduced dissociation of the reagents and solvation of $\mathrm{Cd}^{2+}$ and $\mathrm{WO}_{4}{ }^{2-}$ ions, which are free to move and start the nucleation process. The luminescence properties of the produced nanoparticles were investigated, presenting a broad emission band at around $500 \mathrm{~nm}$, which is comparable to that observed for samples produced using other chemical routes. This result highlights the great potential of the proposed method as a low-cost and time saving process to fabricate luminescent oxide nanoparticles.
\end{abstract}

\section{Introduction}

Recent advances in synthesis technologies demonstrate that the use of microwave radiation is convenient as heating method to obtain nanomaterials in a short reaction time. Further advantages of microwave-based methodology are low cost and low environmental damage $[1,2]$. The fundamental requirement is that solvent and/or reagents employed have the ability to absorb microwave energy in order to convert it into heat [3]. The thermal effects provided by microwaves are consequence of the particle interactions with the electromagnetic field, involving processes of dipolar polarization and ionic conduction $[3,4]$. Such unique feature cannot be achieved using conventional heating, and it provides uniform heating that improves the yield and reproducibility of the route $[3,5]$.

Microwave-assisted hydrothermal method combines the advantages of fast heating and high-pressure reaction. Hydrothermal processes can promote the formation of crystalline products at temperatures much lower than those required in conventional synthesis [6-8]. The combination of microwave heating and hydrothermal environment typically enhances the kinetics of reactions by 1-2 orders of magnitude, saving time and energy [9-11].

Tungstate materials are studied due to their potential application in different areas including catalysis, magnetic devices, humidity sensors, photonics, and scintillators [12, 13]. Conventional solid-state synthesis of $\mathrm{CdWO}_{4}$ departs from metal oxides, which are grinded and heated up to $1000^{\circ} \mathrm{C}$ for several hours $[5,14]$. Alternatively, sol-gel, sonochemical, solvothermal, and conventional hydrothermal syntheses are reported to produce $\mathrm{CdWO}_{4}$ nanoparticles [1522] but it is important to say that in all these works the precursors were either alcoxides or metal salts. Few reports can be found in literature about the microwave-assisted hydrothermal synthesis of $\mathrm{CdWO}_{4}$ [23-25], but they make use of metal salts and organic solvents or surfactants such as toluene and PEG-1000. To our knowledge, microwavehydrothermal method using precursor oxides in aqueous solution was not reported up to the moment to produce nanostructured $\mathrm{CdWO}_{4}$. In the present work, this route was successfully used, satisfying the demand for simplification and improvement of the methodology. The proposed method is also advantageous since it does not require the use of 
surfactants or $\mathrm{pH}$ modifiers, consequently avoiding waste generation. In this work, the mechanism of nucleation and crystal growth, as well as the structural and optical characterization of the produced samples, is presented and discussed.

\section{Materials and Methods}

$\mathrm{CdWO}_{4}$ polycrystalline samples were produced via microwave-assisted hydrothermal method using cadmium oxide and tungsten oxide as precursors. In a typical experiment, $2 \mathrm{mmol}$ of $\mathrm{CdO}$ (Vetec, 99.5\%) and $2 \mathrm{mmol}$ of $\mathrm{WO}_{3}$ (Fluka, $99.9 \%$ ) were homogenized in an agate mortar and placed into a Teflon autoclave filled with $50 \mathrm{ml}$ of distilled water. Then, the autoclave was sealed and placed in the microwave system (Brazilian patent 2008-PI0801233-4) with frequency of $2.45 \mathrm{GHz}$ and maximum power of $800 \mathrm{~W}$. Reactions were conducted at $120^{\circ} \mathrm{C}$ under autogenous pressure $(\sim$ $1.5 \mathrm{kgf} / \mathrm{cm}^{2}$ ) for $20 \mathrm{~min}$ and $60 \mathrm{~min}$. After cooling down to room temperature, the resulting product was collected by centrifugation and dried at $60^{\circ} \mathrm{C}$ in air. Due to CdO losses during heating, synthesis was also performed varying the amount of this reagent to $2.2 \mathrm{mmol}, 2.4 \mathrm{mmol}$, and $2.6 \mathrm{mmol}$,

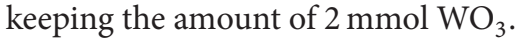

Crystalline phases obtained were characterized by X-ray diffraction (XRD) in a Rigaku Ultima+ 2000/PC using K $\alpha$ radiation from Co. The data were acquired in step-scan mode, in the $2 \theta$ range from $10^{\circ}$ to $80^{\circ}$ with steps of $0.02^{\circ}$ and acquisition time of $10 \mathrm{~s}$. The composition of the samples was determined from energy dispersive X-ray (EDX) spectroscopy measured with Shimadzu EDX-7000 equipment, in semiquantitative mode. Transmission electron microscopy (TEM) images were acquired with Jeol JEM-1400Plus microscope (accelerating voltage of $120 \mathrm{kV}$ ) to evaluate the size and morphology of the powders. Photoluminescence (PL) measurements were performed in an ISS PC1TM spectrofluorimeter that uses a $300 \mathrm{~W}$ Xenon lamp as excitation source, with fixed excitation wavelength at $292 \mathrm{~nm}$. X-ray excited optical luminescence (XEOL) was measured while the samples were under excitation of X-rays emitted by a Co target tube operating at $40 \mathrm{kV} / 40 \mathrm{~mA}$. Both PL and XEOL were collected using an Ocean Optics HR2000 spectrometer (resolution of $0.5 \mathrm{~nm}$ ) and all measurements were performed at room temperature.

\section{Results and Discussion}

XRD patterns of polycrystalline samples prepared using metal oxides in stoichiometric proportion are presented in Figure 1. One can observe that the products obtained by the proposed route are predominantly $\mathrm{CdWO}_{4}$ with monoclinic structure (PDF \#00-088-0181). The temperature of $120^{\circ} \mathrm{C}$ is significantly lower than that used for solid-state synthesis of this scintillator [14, 27]. Furthermore, Figure 1(a) shows that $\mathrm{CdWO}_{4}$ is successfully formed for reaction times as short as $20 \mathrm{~min}$, due to the efficiency of microwave radiation in promoting rapid reactions. Small amount of $\mathrm{WO}_{3}(\mathrm{PDF}$ \#00-083-0950) was detected as secondary phase, indicating that $\mathrm{Cd}$ losses can occur during synthesis, possibly by

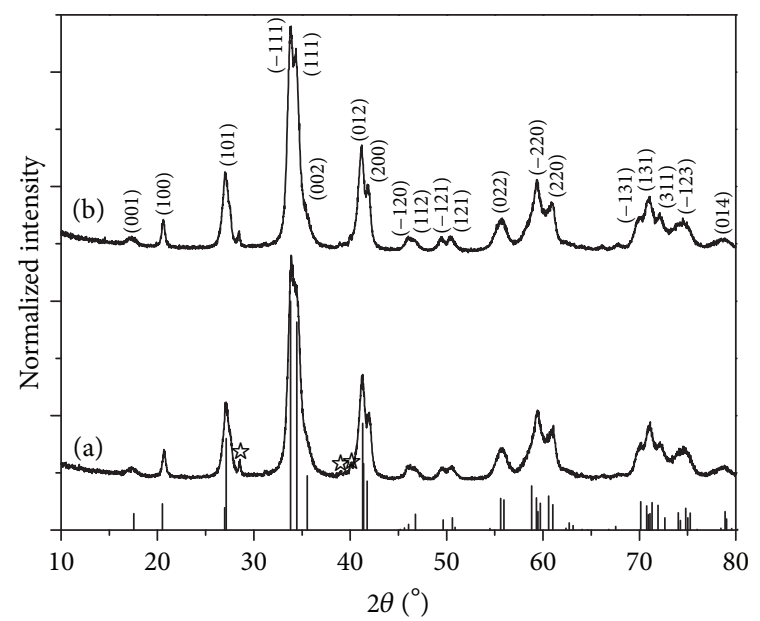

FIGURE 1: XRD patterns of the samples prepared with reagents in stoichiometric proportion, varying the time of synthesis: (a) $20 \mathrm{~min}$ and (b) $60 \mathrm{~min}$. Bars represent the wolframite structure of $\mathrm{CdWO}_{4}$ (some crystalline planes are indexed according to [26]) and symbols correspond to $\mathrm{WO}_{3}$.

volatilization or by dispersion into the water as $\mathrm{Cd}(\mathrm{OH})_{2}$ $[28,29]$. Figure 1(b) corresponds to the nominally stoichiometric sample synthesized during $60 \mathrm{~min}$, indicating that no noticeable change in crystalline structure was detected for longer synthesis times. From the full width at half maximum (FWHM) of the (100) diffraction peak and using Scherrer formula [30], it was possible to estimate the crystallite size D:

$$
D=\frac{K \cdot \lambda}{\beta \cdot \cos \theta_{B}},
$$

where $K$ is the shape coefficient (in this work, $K=0.9$ ), $\lambda$ is the X-ray wavelength $(0.179 \mathrm{~nm}$ for Co target $), \beta$ is the line broadening, and $\theta_{B}$ is the Bragg angle, in radians. The average crystalline sizes were about $23 \pm 1 \mathrm{~nm}$ and $24 \pm 1 \mathrm{~nm}$ for the sample produced with reaction time of 20 and $60 \mathrm{~min}$, respectively.

In order to compensate the Cd loss, samples were produced departing from precursors homogenized with excess CdO. Figure 2 presents XRD patterns for the samples synthesized during $60 \mathrm{~min}$ using 10\% (2.2 mmol), 20\% (2.4 mmol), and $30 \%(2.6 \mathrm{mmol})$ of $\mathrm{CdO}$ excess. The results point to the presence of monoclinic $\mathrm{CdWO}_{4}$ and lower proportions of $\mathrm{WO}_{3}$ phase. The amount of $\mathrm{WO}_{3}$ was $8 \%$ for the sample prepared with reagents in stoichiometric proportion, as estimated from the XRD data using Match! software [31]. When $\mathrm{CdO}$ excess of 10,20 , or $30 \%$ was used, the amount of $\mathrm{WO}_{3}$ phase decreased to $7 \%, 6.5 \%$, and $6 \%$, respectively. Nevertheless, one must notice that the diffraction peaks of $\mathrm{CdWO}_{4}$ become broader with increasing the amount of cadmium precursor (see Figure 2) and such behavior can be related to modifications in the microstructure of these samples, as it will be discussed later in this paper. From the evaluation using (1), a slight decrease was found of the crystalline sizes in relation to that of samples produced without excess of $\mathrm{CdO}$. The results were $21 \pm 1 \mathrm{~nm}, 19 \pm 1 \mathrm{~nm}$, 
TABLE 1: EDX results for atomic composition of the samples.

\begin{tabular}{|c|c|c|c|c|}
\hline Samples & Amount of $\mathrm{CdO}$ & Time of synthesis & $\mathrm{Cd}$ & $\mathrm{W}$ \\
\hline \multirow{2}{*}{ Without using excess of Cd } & $2 \mathrm{mmol}$ & $20 \mathrm{~min}$ & $40 \%$ & $60 \%$ \\
\hline & $2 \mathrm{mmol}$ & $60 \mathrm{~min}$ & $40 \%$ & $60 \%$ \\
\hline \multirow{3}{*}{ Using excess of Cd } & $2.2 \mathrm{mmol}$ & $60 \mathrm{~min}$ & $42 \%$ & $58 \%$ \\
\hline & $2.4 \mathrm{mmol}$ & $60 \mathrm{~min}$ & $44 \%$ & $56 \%$ \\
\hline & $2.6 \mathrm{mmol}$ & $60 \mathrm{~min}$ & $46 \%$ & $54 \%$ \\
\hline
\end{tabular}

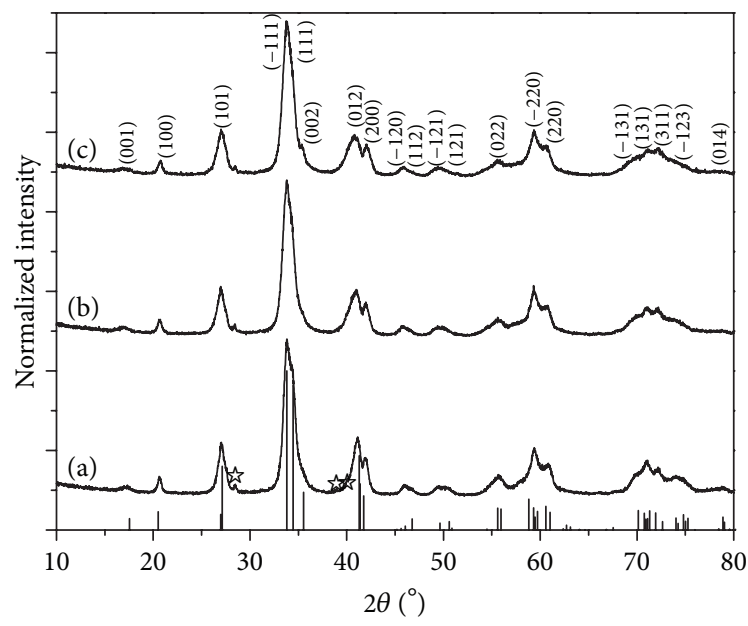

FIGURE 2: XRD patterns of the samples prepared with reagents out of stoichiometric proportion: (a) $10 \%$ excess of CdO, (b) $20 \%$ excess of $\mathrm{CdO}$, and (c) $30 \%$ excess of $\mathrm{CdO}$. Bars represent the wolframite structure of $\mathrm{CdWO}_{4}$ (some crystalline planes are indexed according to [26]) and symbols correspond to $\mathrm{WO}_{3}$.

and $19 \pm 1 \mathrm{~nm}$ when the excess amount of this precursor was 10,20 , and $30 \%$, respectively.

Further investigations about composition of these products were performed through EDX. Results are presented in Table 1. Only Cd and W elements were found in the composition of the samples. Therefore, the level of impurities can be considered negligible. The percentage of $\mathrm{O}$ atoms could not be measured due to experimental limitations of the equipment. This means that, considering only $\mathrm{Cd}$ and $\mathrm{W}$, $\mathrm{CdWO}_{4}$ stoichiometric samples should contain $50 \%$ of each of these elements. However, for all samples synthesized using $\mathrm{CdO}$ and $\mathrm{WO}_{3}$ precursors in stoichiometric proportion, a deficiency of Cd atoms was found. The composition of $60 \%$ $\mathrm{W}$ and $40 \% \mathrm{Cd}$ is the same for synthesis times of 20 and $60 \mathrm{~min}$, indicating a nondependence of the reaction time on stoichiometry, at least for the time intervals used in the present work. One can notice that the amount of $\mathrm{Cd}$ deficiency $(10 \%)$ is in agreement with the XRD results, which pointed out approximately $8 \%$ of secondary phase $\mathrm{WO}_{3}$. Accordingly, for the samples prepared using CdO excess, the deviation from stoichiometry decreased to $8 \%, 6 \%$, and $4 \%$ (see Table 1). These values are also consistent with those obtained from XRD and corroborate that secondary phase of $\mathrm{WO}_{3}$ formed during the hydrothermal reaction can be controlled.
Particle morphology was investigated using TEM, and some of the images obtained are presented in Figure 3. The micrographs show that $\mathrm{CdWO}_{4}$ powders prepared with reagents in stoichiometric proportion at $120^{\circ} \mathrm{C}$ for $20 \mathrm{~min}$ exhibit nanorods with widths of $24 \pm 9 \mathrm{~nm}$ and lengths of 260 $\pm 47 \mathrm{~nm}$, whereas, for reaction time of $60 \mathrm{~min}$, particles with widths of $11 \pm 3 \mathrm{~nm}$ and lengths of $98 \pm 33 \mathrm{~nm}$ were observed. Therefore, Figures 3(a) and 3(b) indicate that the aspect ratio (or length-to-diameter ratio) is very similar despite increasing the time of synthesis. The nanorod formation is attributed to a preferred growth along the (100) direction at the expense of individual crystallites [32, 33], and it is an interesting result of this work, considering that no template or surface passivation was used in the production, as related in previous reports. The morphology obtained in this work is consistent with those reported for the synthesis of $\mathrm{CdWO}_{4}$ departing from metal salts combined to PEG1000 surfactant [24], indicating that $\mathrm{CdWO}_{4}$ preferably grows into the nanorod morphology, regardless of the nature of the chemical reagents. Also, from Figures 3(a) and 3(b), it can be observed that samples prepared with reaction time of $60 \mathrm{~min}$ present smoother particle surface. This effect is probably due to Ostwald ripening or better crystallization of the particles hydrothermalized for longer times, although negligible influence of reaction time on the phase formation was detected from XRD data. Figures 3(c) and 3(d) correspond to samples produced with Cd excess of $20 \%$ and $30 \%$, respectively (synthesis times of $60 \mathrm{~min}$ in both cases). In these micrographs, the formation of thinner particles, which tend to agglomerate into bundles, can be noticed. Respectively, their sizes were found to be of $10 \pm 2 \mathrm{~nm}$ width and $161 \pm$ $27 \mathrm{~nm}$ length or $10 \pm 2 \mathrm{~nm}$ width and $146 \pm 50 \mathrm{~nm}$ length, which point to higher aspect ratios in comparison to that of samples produced without excess of Cd. Such microstructure is less common but can be related to small changes in the experimental procedure that originally produced nanorods $[20,34]$.

About the growth mechanism of $\mathrm{CdWO}_{4}$ using oxide precursors under microwave-hydrothermal conditions, some assumptions can be made. Initially, the microwave radiation promotes a superheating of the water as well as of the $\mathrm{CdO}$ and $\mathrm{WO}_{3}$ particles. These reagents undergo a dissociation so that $\mathrm{Cd}^{2+}$ and $\mathrm{WO}_{4}{ }^{2-}$ are produced and solvated by $\mathrm{H}_{2} \mathrm{O}$ molecules. This means that the partial negative charges of $\mathrm{H}_{2} \mathrm{O}$ electrostatically attract $\mathrm{Cd}^{2+}$ ions whereas the positive charges attract $\mathrm{WO}_{4}{ }^{2-}$. Then, free ions are able to move by the action of the radiation, and collisions occur. In such conditions, nucleation takes place in a very short time 


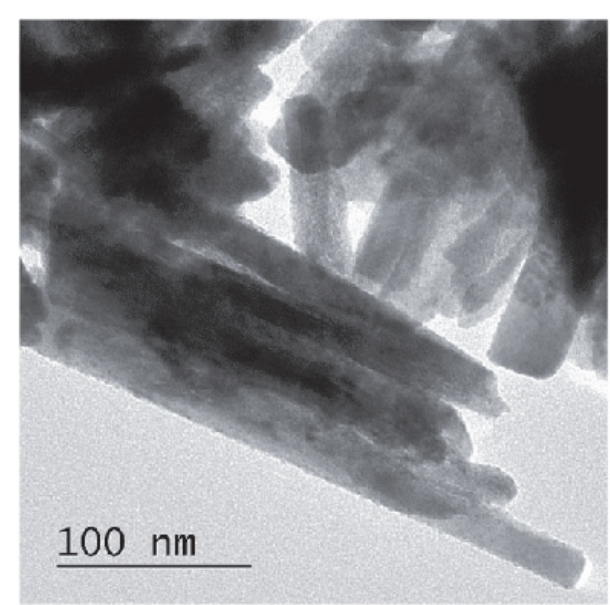

(a)

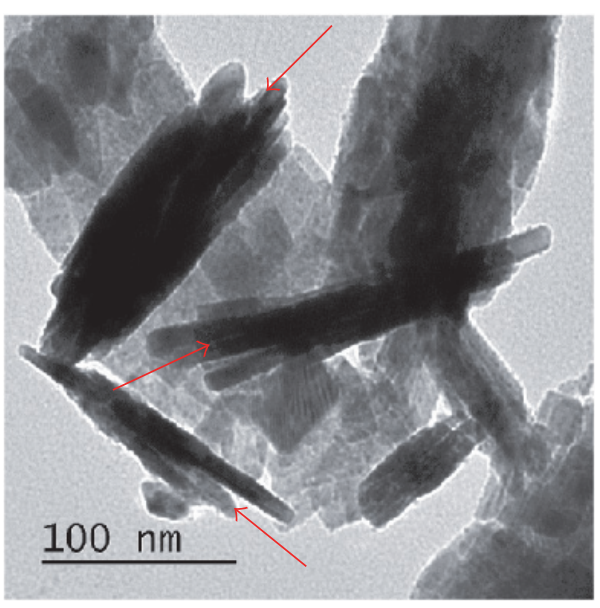

(c)

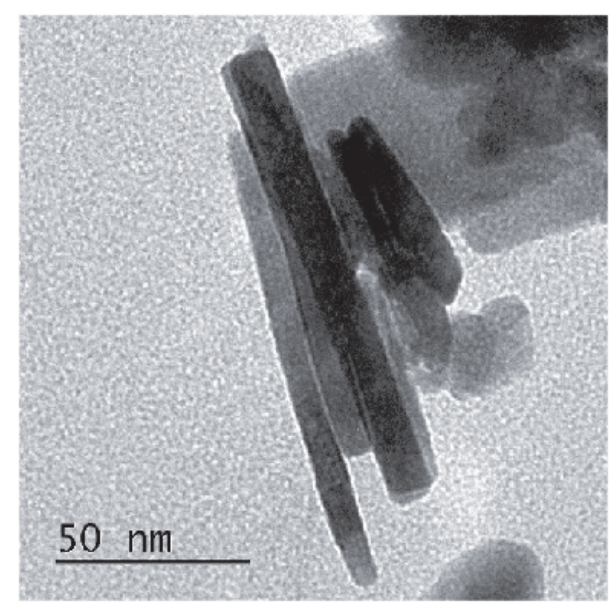

(b)

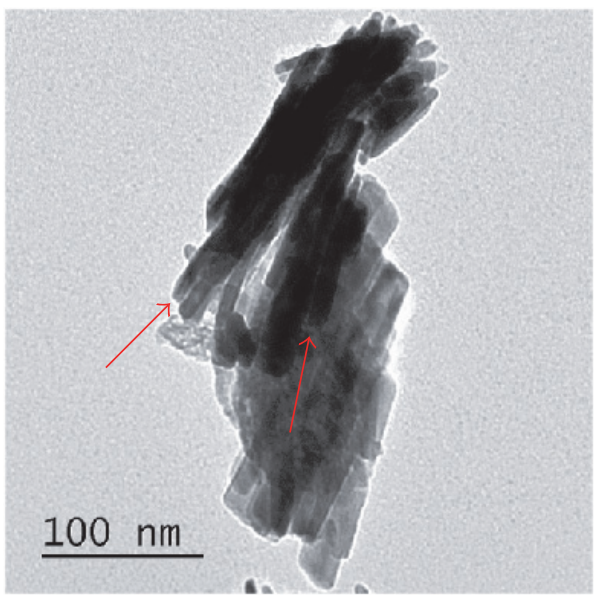

(d)

FIGURE 3: TEM images of $\mathrm{CdWO}_{4}$ nanorods produced with varied conditions: using reagents in stoichiometric proportion, soaking time of (a) $20 \mathrm{~min}$ and (b) $60 \mathrm{~min}$, or using Cd excess of (c) $20 \%$ and (d) $30 \%$. Some nanofiber bundles are indicated by red arrows.

and thus leads to the production of particles in nanoscale regime $[35,36]$. In the present case, $\mathrm{CdWO}_{4}$ crystals are crystallographic oriented and self-assembled into nanorod or nanofiber morphologies.

PL and XEOL emission spectra are presented in Figures 4 and 5, respectively. Upon excitation of either UV or X-ray, the samples presented a broad band emission with maximum at $500 \mathrm{~nm}$, characteristic of $\mathrm{CdWO}_{4}$ and attributed to charge transfer transitions between $\mathrm{W}^{6+}$ and $\mathrm{O}^{2-}$ ions within the tungstate molecular units $\mathrm{WO}_{6}[37,38]$. The luminescence profile was basically the same for reaction times of $60 \mathrm{~min}$ (presented in Figures 4 and 5) or $20 \mathrm{~min}$ (not presented). The total light output was obtained from the area under each curve, with the highest light output set as 100. These results are presented in the inset of Figures 4 and 5, as function of $\mathrm{Cd} / \mathrm{W}$ ratio (as determined by EDX) and reaction times. For both excitation sources, it was observed that the light yield increases for longer reaction times, for all stoichiometric proportions used. This is probably due to the higher crystallinity achieved with longer hydrothermal treatments. Similar results were reported for $\mathrm{CdWO}_{4}$ samples produced by conventional hydrothermal synthesis at different conditions $[39,40]$. On the other hand, works in $[23,24]$, about the microwave-assisted method, point to an opposite dependence of PL intensity with the reaction time, demonstrating that in these cases other factors such as particles size and the surface modification by surfactants can influence the luminescence emission.

Figures 4 and 5 show that the most intense emission corresponds to that of nominally stoichiometric samples, where the actual proportion of $\mathrm{Cd} / \mathrm{W}$ is $40 / 60$. This result suggests that the different morphologic features of the samples produced with or without $\mathrm{Cd}$ excess have a strong influence on the light output of the samples. This is probably due to the different effective areas of the particles with rod or fiber morphologies. Despite the fact that $\mathrm{WO}_{3}$ as secondary phase tends to influence negatively the recombination rate of photogenerated charge carriers [41], the role played by microstructure seems to be more crucial for the light output efficiency in this case. In future works, an in-depth study of 


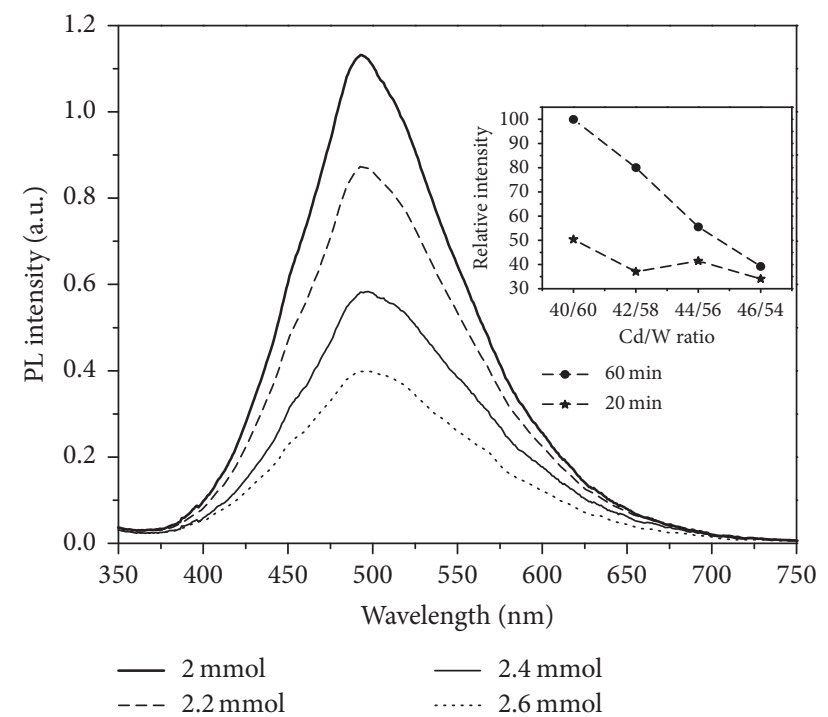

FIGURE 4: PL emission spectra of samples prepared with different cadmium precursor $(\mathrm{CdO})$ amounts and reaction time of $60 \mathrm{~min}$. The inset shows the total light output, for reaction times of $20 \mathrm{~min}$ and $60 \mathrm{~min}$, obtained from the area under the luminescence curves, as function of $\mathrm{Cd} / \mathrm{W}$ atomic ratio, as determined by EDX.

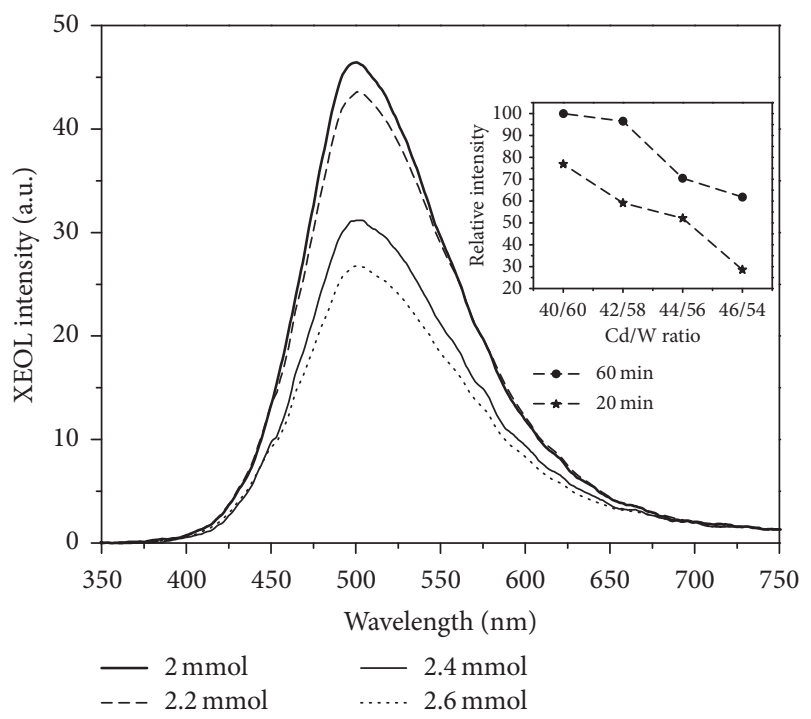

FIGURE 5: XEOL emission spectra of samples prepared with different cadmium precursor $(\mathrm{CdO})$ amounts and reaction time of $60 \mathrm{~min}$. The inset shows the total light output, for reaction times of $20 \mathrm{~min}$ and $60 \mathrm{~min}$, obtained from the area under the luminescence curves, as function of $\mathrm{Cd} / \mathrm{W}$ atomic ratio, as determined by EDX.

surface area and light output should be carried on to establish a quantitative relationship between these two parameters.

\section{Conclusions}

The results presented in this work show the feasibility of using oxide precursors for synthesizing $\mathrm{CdWO}_{4}$ under microwaveassisted hydrothermal conditions. Methodology is conducted at short times and low temperature, being environmentally safe and also saving time and energy. Monoclinic structure and nanorod or bundle morphologies of $\mathrm{CdWO}_{4}$ were obtained, depending on Cd excess used in the synthesis. From the luminescence measurements, it was observed that the microstructure plays an important role in the light output of these scintillators. Samples prepared with excess of CdO precursor in relation to the stoichiometric proportion provided some reduction of the $\mathrm{WO}_{3}$ content in the final product, but they have presented lower luminescence. Nevertheless, increasing the reaction time from 20 to $60 \mathrm{~min}$ favored the crystallization process and such samples have an overall higher efficiency. Different methods to avoid the loss of cadmium and formation of single crystalline phase are under investigation and will be presented in future works.

\section{Competing Interests}

The authors declare that they have no competing interests.

\section{Acknowledgments}

The authors are grateful to the Brazilian agencies CAPES, CNPq, FINEP, FAPITEC, and COPES/UFS for financial support. They also acknowledge CMNano-UFS, Proposal \#85.

\section{References}

[1] X. Hu and J. C. Yu, "Continuous aspect-ratio tuning and fine shape control of monodisperse $\alpha-\mathrm{Fe}_{2} \mathrm{O}_{3}$ nanocrystals by a programmed microwave-hydrothermal method," Advanced Functional Materials, vol. 18, no. 6, pp. 880-887, 2008.

[2] C. S. Lim, "Microwave-assisted synthesis and photoluminesence of $\mathrm{CdWO}_{4}$ by a solid-state metathetic route," Asian Journal of Chemistry, vol. 24, no. 9, pp. 4166-4170, 2012.

[3] I. Bilecka and M. Niederberger, "Microwave chemistry for inorganic nanomaterials synthesis," Nanoscale, vol. 2, no. 8, pp. 1358-1374, 2010.

[4] S. Das, A. K. Mukhopadhyay, S. Datta, and D. Basu, "Prospects of microwave processing: an overview," Bulletin of Materials Science, vol. 32, no. 1, pp. 1-13, 2009.

[5] D. S. Sofronov, E. M. Sofronova, V. V. Starikov et al., "Microwave synthesis of cadmium and zinc tungstates," Journal of Materials Engineering and Performance, vol. 21, no. 11, pp. 2323-2327, 2012.

[6] S. Komarneni, R. Roy, and Q. H. Li, "Microwave-hydrothermal synthesis of ceramic powders," Materials Research Bulletin, vol. 27, no. 12, pp. 1393-1405, 1992.

[7] D. Yue, W. Luo, W. Lu et al., "A facile synthesis and optical properties of bundle-shaped $\mathrm{TbPO}_{4} \cdot \mathrm{H}_{2} \mathrm{O}$ nanorods," Advances in Condensed Matter Physics, vol. 2013, Article ID 673945, 5 pages, 2013.

[8] K. Byrappa, "Hydrothermal growth of polyscale crystals," in Springer Handbook of Crystal Growth, G. Dhanaraj, K. Byrappa, V. Prasad, and M. Dudley, Eds., pp. 599-653, Springer, Berlin, Germany, 2010.

[9] L. S. Cavalcante, J. C. Sczancoski, L. F. Lima Jr. et al., "Synthesis, characterization, anisotropic growth and photoluminescence of $\mathrm{BaWO}_{4}$," Crystal Growth and Design, vol. 9, no. 2, pp. 1002-1012, 2009. 
[10] G. Biasotto, A. Z. Simões, C. R. Foschini, M. A. Zaghete, J. A. Varela, and E. Longo, "Microwave-hydrothermal synthesis of perovskite bismuth ferrite nanoparticles," Materials Research Bulletin, vol. 46, no. 12, pp. 2543-2547, 2011.

[11] M. L. Moreira, G. P. Mambrini, D. P. Volanti et al., "Hydrothermal microwave: a new route to obtain photoluminescent crystalline $\mathrm{BaTiO}_{3}$ nanoparticles," Chemistry of Materials, vol. 20, no. 16, pp. 5381-5387, 2008.

[12] X. C. Song, X. Cui, W. Z. Huang, Y. Zhang, H. Y. Yin, and Y. F. Zheng, "Photoactivity enhancement of Zn-doped $\mathrm{CdWO}_{4}$ prepared with a hydrothermal method," Materials Science and Engineering B: Solid-State Materials for Advanced Technology, vol. 197, pp. 31-35, 2015.

[13] L. L. Nagornaya, F. A. Danevich, A. M. Dubovik et al., "Tungstate and molybdate scintillators to search for dark matter and double beta decay," IEEE Transactions on Nuclear Science, vol. 56, no. 4, pp. 2513-2518, 2009.

[14] W. Ran, L. Wang, H. Li et al., "Luminescence properties and energy transfer of $\mathrm{CdWO}_{4}: \mathrm{Sm}^{3+}, \mathrm{Bi}^{3+}, \mathrm{M}^{+}(\mathrm{M}=\mathrm{Li}, \mathrm{Na}, \mathrm{K})$ phosphors for white LEDs," Ceramics International, vol. 41, no. 3, pp. 4301-4307, 2015.

[15] L. Nadaraia, N. Jalabadze, R. Chedia, M. Antadze, and L. Khundadze, "Preparation of Tungstate nanopowders by Sol-Gel method," IEEE Transactions on Nuclear Science, vol. 57, no. 3, pp. 1370-1376, 2010.

[16] H. Shang, Y. Wang, B. Milbrath, M. Bliss, and G. Cao, "Doping effects in nanostructured cadmium tungstate scintillation films," Journal of Luminescence, vol. 121, no. 2, pp. 527-534, 2006.

[17] S. M. Hosseinpour-Mashkani and A. Sobhani-Nasab, "A simple sonochemical synthesis and characterization of $\mathrm{CdWO}_{4}$ nanoparticles and its photocatalytic application," Journal of Materials Science: Materials in Electronics, vol. 27, no. 4, pp. 3240-3244, 2016.

[18] A. J. Rondinone, M. Pawel, D. Travaglini, S. Mahurin, and S. Dai, "Metastable tetragonal phase $\mathrm{CdWO}_{4}$ nanoparticles synthesized with a solvothermal method," Journal of Colloid and Interface Science, vol. 306, no. 2, pp. 281-284, 2007.

[19] Y. Wang, X. Guan, L. Li, H. Lin, X. Wang, and G. Li, "Solventdriven polymorphic control of $\mathrm{CdWO}_{4}$ nanocrystals for photocatalytic performances," New Journal of Chemistry, vol. 36, no. 9, pp. 1852-1858, 2012.

[20] Y. Wang, J. Ma, J. Tao et al., "Hydrothermal synthesis and characterization of $\mathrm{CdWO}_{4}$ nanorods," Journal of the American Ceramic Society, vol. 89, no. 9, pp. 2980-2982, 2006.

[21] Y. Wang, J. Ma, J. Tao et al., "Low-temperature synthesis of $\mathrm{CdWO}_{4}$ nanorods via a hydrothermal method," Ceramics International, vol. 33, no. 6, pp. 1125-1128, 2007.

[22] Q. Dai, H. Song, G. Pan et al., "Surface defects and their influence on structural and photoluminescence properties of $\mathrm{CdWO}_{4}: \mathrm{Eu}^{3+}$ nanocrystals," Journal of Applied Physics, vol. 102, no. 5, Article ID 054311, 2007.

[23] L. Hou, L. Lian, L. Zhang, T. Wu, and C. Yuan, "Microwaveassisted interfacial hydrothermal fabrication of hydrophobic $\mathrm{CdWO}_{4}$ microspheres as a high-performance photocatalyst," RSC Advances, vol. 4, no. 5, pp. 2374-2381, 2014.

[24] J. Yan, Y. Shen, R. Cao, and T. Li, "CdWO ${ }_{4}$ nanorods: ultrafast synthesis via a PEG-1000 polymer-assisted enhanced microwave synthesis route and their photoluminescence property," Ceramics International, vol. 40, no. 6, pp. 8081-8085, 2014.
[25] A. S. Vanetsev, Y. V. Orlovskii, V. Nagirnyi et al., “Testing nanocrystalline $\mathrm{CdWO}_{4}$ doped with $\mathrm{Yb}^{3+}$ as a possible downconversion phosphor," Radiation Measurements, vol. 9, pp. 329333, 2016.

[26] M. Daturi, G. Busca, M. M. Borel, A. Leclaire, and P. Piaggio, "Vibrational and XRD study of the system $\mathrm{CdWO}_{4}-\mathrm{CdMoO}_{4}$ ", Journal of Physical Chemistry B, vol. 101, no. 22, pp. 4358-4369, 1997.

[27] S. M. V. Novais, R. S. Da Silva, and Z. S. MacEdo, "Thermally stimulated luminescence of polycrystalline $\mathrm{CdWO}_{4}$ at low temperatures," Journal of Luminescence, vol. 131, no. 7, pp. 12831287, 2011.

[28] J. Yang, Y. Li, S. Liu et al., "Theoretical calculations of Cd isotope fractionation in hydrothermal fluids," Chemical Geology, vol. 391, pp. 74-82, 2015.

[29] Z.-X. Yang, W. Zhong, Y.-X. Yin et al., "Controllable synthesis of single-crystalline $\mathrm{CdO}$ and $\mathrm{Cd}(\mathrm{OH})_{2}$ nanowires by a simple hydrothermal approach," Nanoscale Research Letters, vol. 5, no. 6, pp. 961-965, 2010.

[30] B. D. Cullity, "Diffraction I: directions of diffracted beams," in Elements of X-Rayy Diffraction, pp. 81-106, Addison-Wesley, Philippines, 1978.

[31] H. Putz and K. Brandenburg, Match! Phase Identification From Powder Diffraction Data, Version 3.2.1, 2016.

[32] D. Ye, D. Li, W. Zhang et al., "A new photocatalyst $\mathrm{CdWO}_{4}$ prepared with a hydrothermal method," Journal of Physical Chemistry C, vol. 112, no. 44, pp. 17351-17356, 2008.

[33] Y. Wang, L. Yang, Y. Wang, X. Xu, and X. Wang, "Controllable synthesis of $\mathrm{CdWO}_{4}$ nanorods and nanowires via a surfactantfree hydrothermal method," Journal of the Ceramic Society of Japan, vol. 120, no. 1402, pp. 259-261, 2012.

[34] H.-L. Wang, X.-D. Ma, X.-F. Qian, J. Yin, and Z.-K. Zhu, "Selective synthesis of $\mathrm{CdWO}_{4}$ short nanorods and nanofibers and their self-assembly," Journal of Solid State Chemistry, vol. 177, no. 12, pp. 4588-4596, 2004.

[35] K. P. F. Siqueira, R. L. Moreira, M. Valadares, and A. Dias, "Microwave-hydrothermal preparation of alkaline-earth-metal tungstates," Journal of Materials Science, vol. 45, no. 22, pp. 6083-6093, 2010.

[36] L. S. Cavalcante, V. M. Longo, J. C. Sczancoski et al., "Electronic structure, growth mechanism and photoluminescence of $\mathrm{CaWO}_{4}$ crystals," CrystEngComm, vol. 14, no. 3, pp. 853-868, 2012.

[37] M. M. Chirila, K. T. Stevens, H. J. Murphy, and N. C. Giles, "Photoluminescence study of cadmium tungstate crystals," Journal of Physics and Chemistry of Solids, vol. 61, no. 5, pp. 675$681,2000$.

[38] V. Nagirnyi, M. Kirm, A. Kotlov, A. Lushchik, and L. Jönsson, "Separation of excitonic and electron-hole processes in metal tungstates," Journal of Luminescence, vol. 102-103, pp. 597-603, 2003.

[39] M. Hojamberdiev, R. Kanakala, O. Ruzimuradov, Y. Yan, G. Zhu, and $\mathrm{Y}$. Xu, "Besom-like CdWO4 structures composed of singlecrystalline nanorods grown under a simple hydrothermal process in ultra-wide pH range," Optical Materials, vol. 34, no. 11, pp. 1954-1957, 2012.

[40] W. Tong, L. Li, W. Hu, T. Yan, and G. Li, "Systematic control of monoclinic $\mathrm{CDWO}_{4}$ nanophase for optimum photocatalytic activity," Journal of Physical Chemistry C, vol. 114, no. 3, pp. 15121519, 2010. 
[41] I. Aslam, C. Cao, M. Tanveer et al., "A novel Z-scheme $\mathrm{WO}_{3} / \mathrm{CdWO}_{4}$ photocatalyst with enhanced visible-light photocatalytic activity for the degradation of organic pollutants," RSC Advances, vol. 5, no. 8, pp. 6019-6026, 2015. 

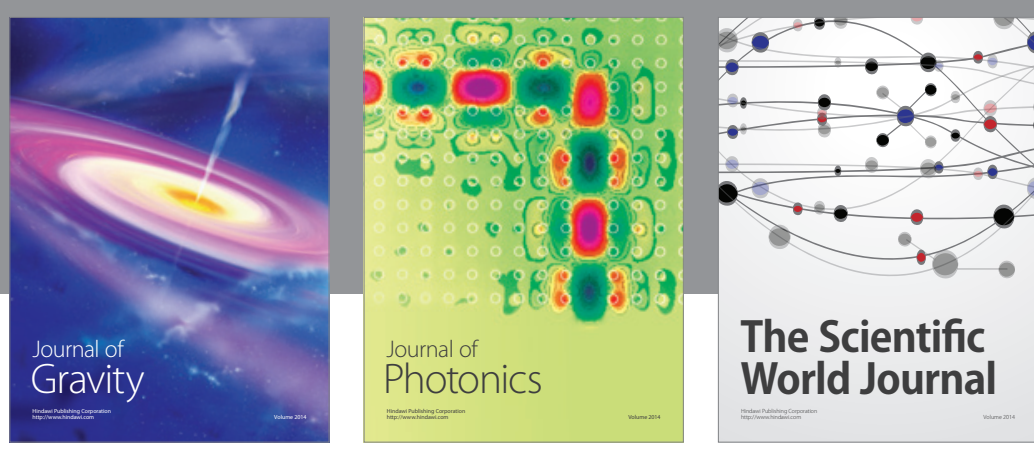

The Scientific World Journal
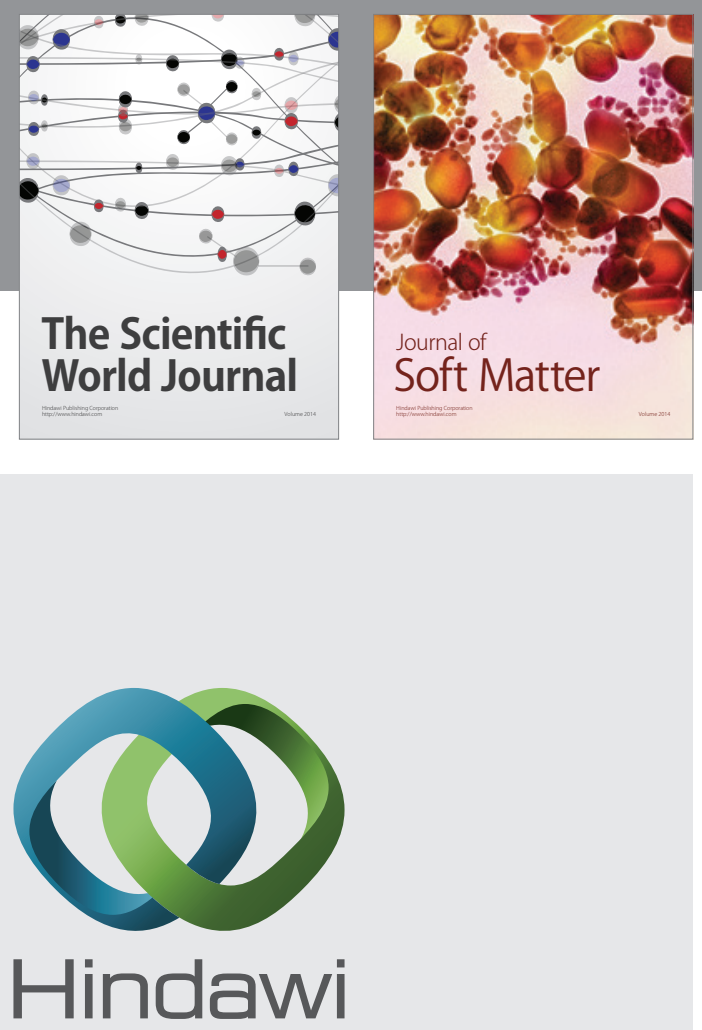

Submit your manuscripts at

http://www.hindawi.com

nternational Journal of

Statistical Mechanics
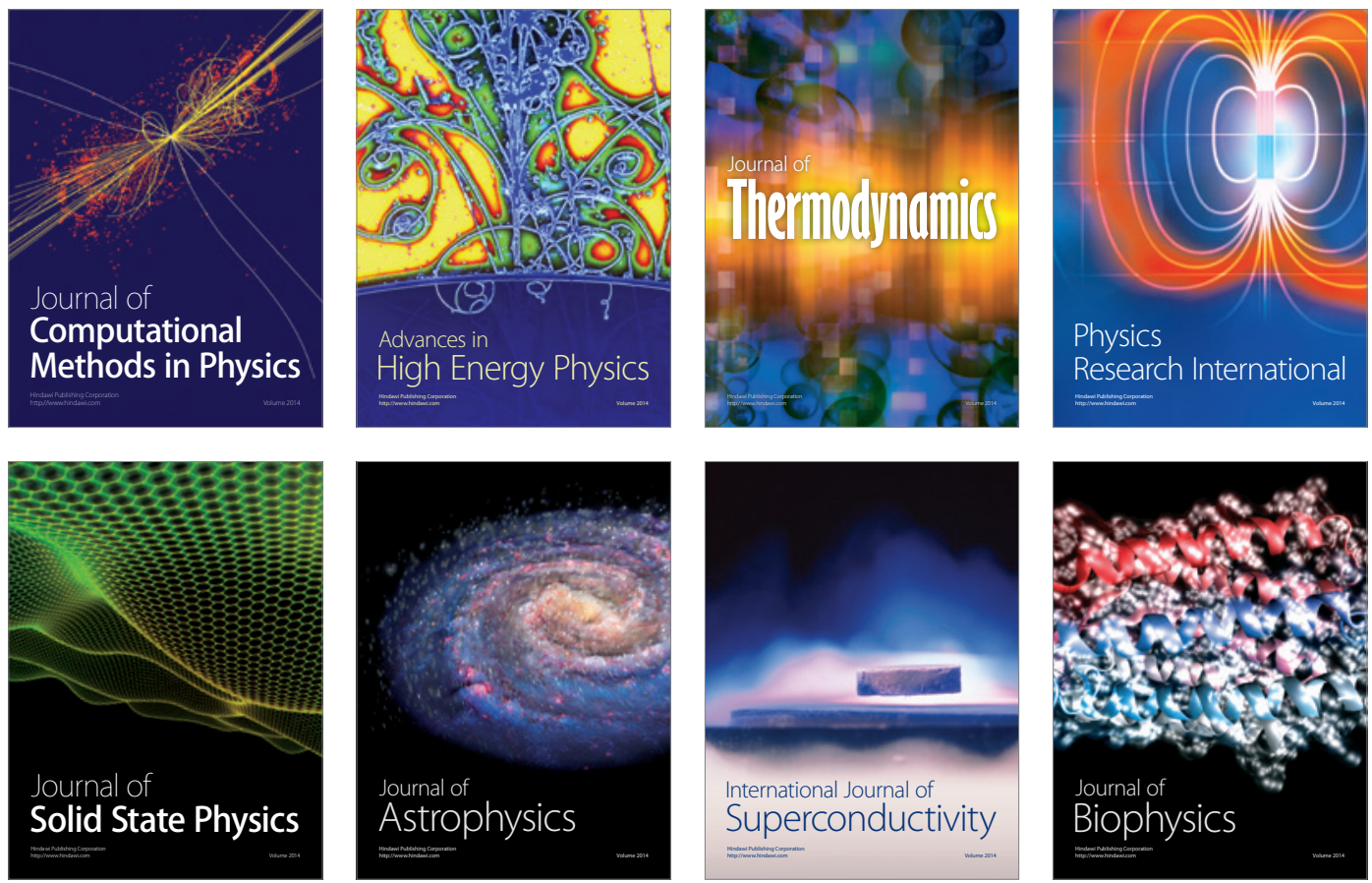
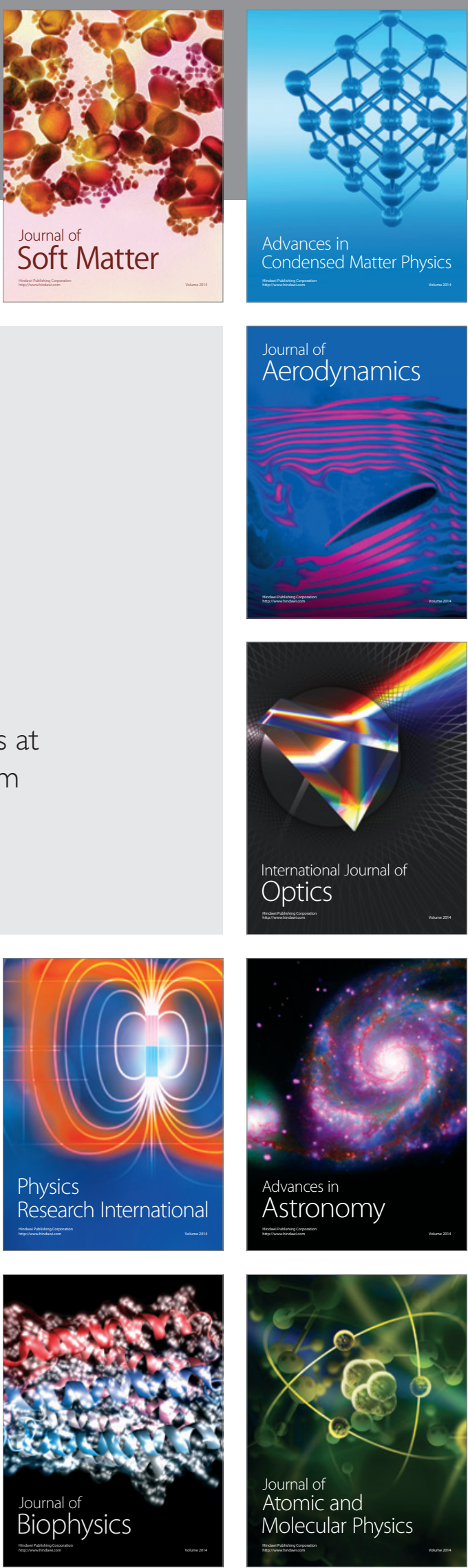